\title{
Density-Viscosity Measurement Cell for Electrodynamic-Acoustic Resonators
}

Frieder Lucklum, Bernhard Jakoby

Institute for Microelectronics and Microsensors, Johannes Kepler University Linz, Altenberger Str. 69, A-4040 Linz, Austria

E-mail: frieder.lucklum@jku.at

\begin{abstract}
Miniaturized physical sensors for precise density and viscosity analysis are required as supplement and replacement for complex and expensive laboratory instruments. Using miniature mechanical resonators as transducers, one can greatly reduce the necessary liquid volume, measurement time, and complexity of the experimental setup. A newly developed miniature, modular measurement setup is investigated over a large viscosity range and at different temperatures. The inexpensive measurement cell utilizes multiple PCB layers, Parafilm or Teflon sealing, and electrodynamically excited metallic resonator elements operating at low frequency shear vibration modes.
\end{abstract}

\section{Introduction}

Liquid properties as density, compressibility, and viscosity are important measurement parameters for the process industry, analytical chemistry, biochemistry, and bioanalytics. Standard laboratory instruments are very precise, but usually highly complex, expensive, and require large amounts of analyte and often long measurement times. Miniaturized acoustic sensors can act as supplement and replacement for these instruments [1].

Electromagnetic transduction of mechanical resonances has been established as a low cost alternative to piezoelectric or capacitive methods with unique advantages in terms of suitable materials, vibration mode flexibility, and simplicity of the excitation setup. We have previously demonstrated different successful approaches to electromagnetic-acoustic sensors. These devices include suspended beams and plates, remotely excited silicon membranes and metal plates, and clamped polymer membranes, e.g. [2-6].

The electrodynamic excitation of a mechanical vibration is based on the generation of Lorentz forces in a resonator element. This is achieved by impressing or inducing an alternating electrical current in a conductive layer of the resonator and placing the device in a static magnetic field, e.g., supplied by external permanent magnets. According to the Lorentz force law, this leads to alternating forces resulting in a vibration of the element in a direction perpendicular to current and magnetic field at the frequency of the driving current. When the driving frequency coincides with an eigenfrequency of the sensor element, mechanical resonance is achieved, leading to a great increase of the vibration amplitude. For electrodynamic detection, the movement of the resonator in the magnetic field leads to the induction of a secondary voltage, which is used as a direct measure for the mechanical vibration and resonance. Electromagnetic-acoustic resonant transducers are thus suited as sensors similar to established acoustic sensors such as piezoelectric (e.g. quartz crystal) resonators, cantilever devices, or capacitive transducers [7-15].

\section{Design and Fabrication}

In this work, Lorentz forces are generated by directly impressing an alternating current across suspended plate resonators made of different conductive substrates (nickel-zinc, aluminum, copper, brass, stainless steel) with a superposed, perpendicular external magnetic field of $0.72 \mathrm{~T}$ flux density supplied by $\mathrm{NdFeB}$ magnets. The measurement cell was developed as a modular design for easy assembly and disassembly, milled into inexpensive PCB material, interspaced with Parafilm ${ }^{\circledR}$ (thermoplastic) or Teflon ${ }^{\circledR}$ (PTFE) sheets, and electrically contacted and fluidically sealed by tightly screwing the different modules together. This inexpensive design allows for quick cleaning and exchange of the sensor element. 


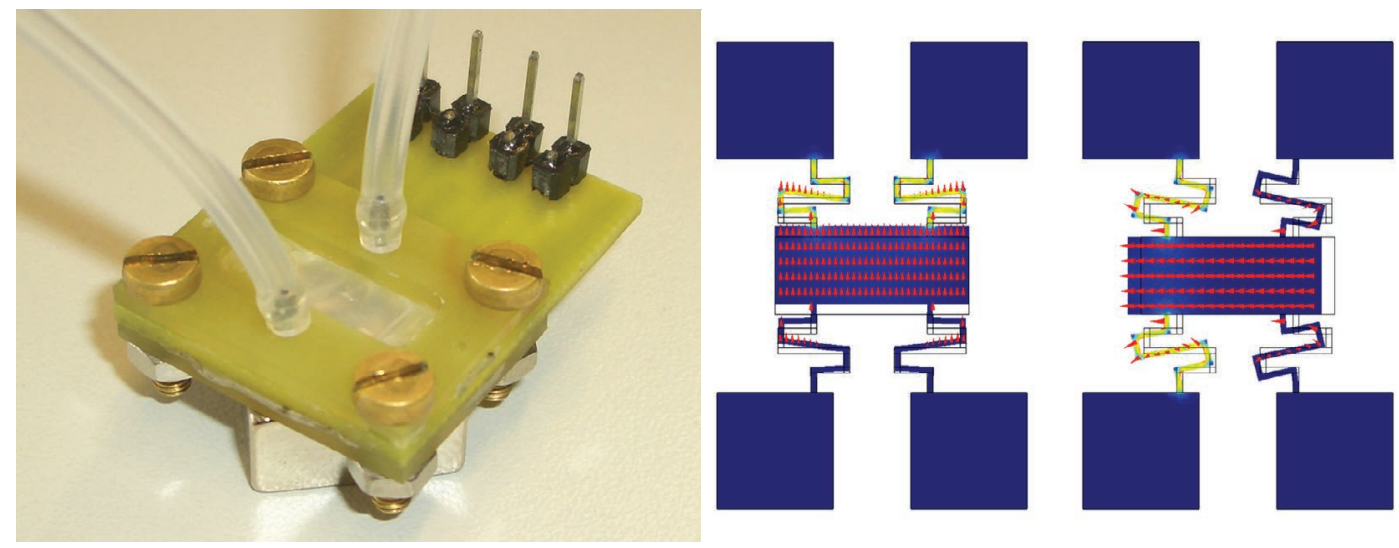

Fig. 1: Fabricated flow-through cell in PCB with permanent magnet below and cavity for second magnet on top (left), and FEM excitation analysis for current density (colored regions) and displacement (arrows) for vertical and horizontal in-plane vibration at $3.8 \mathrm{kHz}$ and $5.7 \mathrm{kHz}$, respectively (right).

The suspended plate resonators have been fabricated by standard photolithography and etching, milling, and laser cutting of thin metallic sheets. Fluidic inlet and outlet are connected to standard capillaries through the top PCB layer. Only small amounts of liquids (on the order of $100 \mu \mathrm{l}$ ) are required. The test fluid can be quickly exchanged with a combination of solvent cleaning and air flow drying, and the whole device can also be easily disassembled for extensive cleaning or replacement of the resonator. The dimensions of the complete device are $2.5 \mathrm{~cm}$ by $3 \mathrm{~cm}$ by $1 \mathrm{~cm}$. The resonator itself is electrically operated as a two-port device, where the excitation can be applied to any two contact pads, while the other two are connected to a network analyzer to measure the voltage induced by mechanical motion. The excitation current can thus flow across the plate in a horizontal, vertical and diagonal fashion, resulting in vertical, horizontal and diagonal forces, respectively (Fig. 1). The resulting in-plane vibration exerts a shear stress at the solid-liquid interface on the top and bottom faces of the resonator. This leads to an evanescent shear wave penetrating the liquid, which is highly sensitive to the density-viscosity product $\rho \eta$, yielding shifts of the resonance frequency $f$ and changes in the quality factor $Q$.

The sensor setup was connected to an Agilent E5061B network analyzer for gain-phase measurements. The DC impedance of the resonator elements was in the range of a few Ohms. The excitation path was connected to reflection port and RF source at $0 \mathrm{dBm}$ nominal power at $50 \Omega$, while the induction path of the resonator was connected to the transmission port of the network analyzer. To decouple parasitic effects of cables, crosstalk, offset, etc., and to amplify driving current and induced voltage, two low frequency transformers (Coilcraft Europe Ltd., UK) with winding ratios of 1:8 and 1:16, respectively, were attached to the connection pins. No additional averaging or smoothening was necessary. For temperature control and stabilization the device was placed into a climate control chamber (Weiss WKL 100).
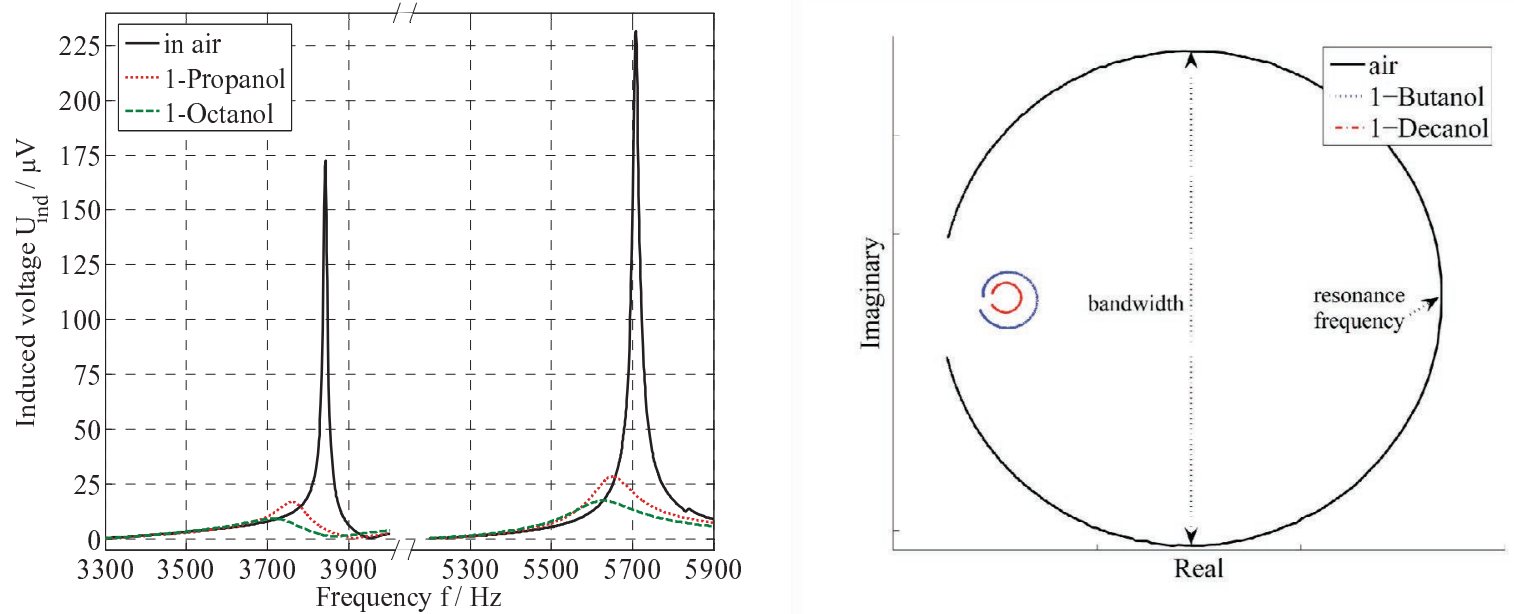

Fig. 2: Measured induced voltage in air and liquids for vertical mode at $3.8 \mathrm{kHz}$ and horizontal mode at $5.7 \mathrm{kHz}$, corresponding to Fig. 1 (left), and exemplary locus plot for horizontal mode (right). 


\section{Experimental Results}

From the locus plot of the transfer function in the vicinity of the resonance frequency, we evaluate the quality factor and frequency shift in different liquids as a function of the density-viscosity product [7, 11]. Figure 2 shows two exemplary measurements of a vertical and horizontal vibration corresponding to the FEM analysis in figure 1, as frequency spectrum and locus plot, respectively. We analyzed liquids over a wide range of viscosities, including de-ionized (DI) water, primary alcohols, and oil viscosity standards up to $400 \mathrm{mPas}$. Reference values for density and viscosity of these test liquids were measured with a high precision lab viscosimeter (Anton Paar SVM 3000) in parallel to the resonance sensor measurements. The test liquids were injected with a microliter pipette. After each liquid, the test chamber was flushed with appropriate cleaning solvent (benzine, isopropanol, acetone), airflow dried, and a reference measurement in air was made. The measurement cell was only disassembled after a complete measurement run.

Shown in figure 3 are the measurement results for a nickel-zinc alloy device of $100 \mu \mathrm{m}$ thickness with linear anchor springs operating in a shear horizontal mode of vibration around $9 \mathrm{kHz}$. Each measurement was repeated ten times, the mean values and standard deviation used for the plots below.

The Q-factor, which decreases from about 250 in air to 10-80 in liquids, displays a continuous, non-linear behavior. Only water slightly deviates from this behavior, which can be attributed to the larger difference in density and spurious out-of-plane motion at the edges appearing in this specific experiment, because out-of-plane motion is particularly sensitive to density $[16,17]$. The still considerable quality factor of the S200 oil viscosity standard leads to the prediction that these resonator sensors are also suitable for even higher viscosities. However, the handling of such highly viscous liquids becomes increasingly difficult with the current miniaturized design, especially the filling and flushing of the measurement cell due to the small dimensions of cell, inlets, and capillaries. The standard deviation of the measured quality factor is approximately $1-2 \%$. The frequency shift displays a nearly linear relationship to the square root of density-viscosity. However, especially for liquids of higher viscosities, establishing an appropriate reference measurement in air becomes difficult, due to problems with complete cleaning and drying of the resonator element. Liquid and solvent residue strongly affect the air measurement due to adsorption at the resonator surface, while during measurement in other liquids, these trace substances become negligible. The average standard deviation of the frequency shift is $1 \%$.

A primary issue of viscosity measurements is the strong dependence of the measurand on temperature. Therefore, we additionally evaluated the temperature behavior of our sensor setup in air and liquids over a wider temperature range. Of concern is the influence of temperature not only on viscosity, but also on the mechanical, elastic properties of the resonator material, on the clamping boundary conditions due to changes in geometry, and also on the electrical properties of the contact between PCB and resonator.

For the measurements in air shown in figure 4, a stainless steel element of $200 \mu \mathrm{m}$ thickness was used. For sealing, Teflon instead of Parafilm was employed, allowing for a larger temperature range from $-10^{\circ} \mathrm{C}-100^{\circ} \mathrm{C}$ and less deviation due to changes in the sealing and boundary conditions. The resonance
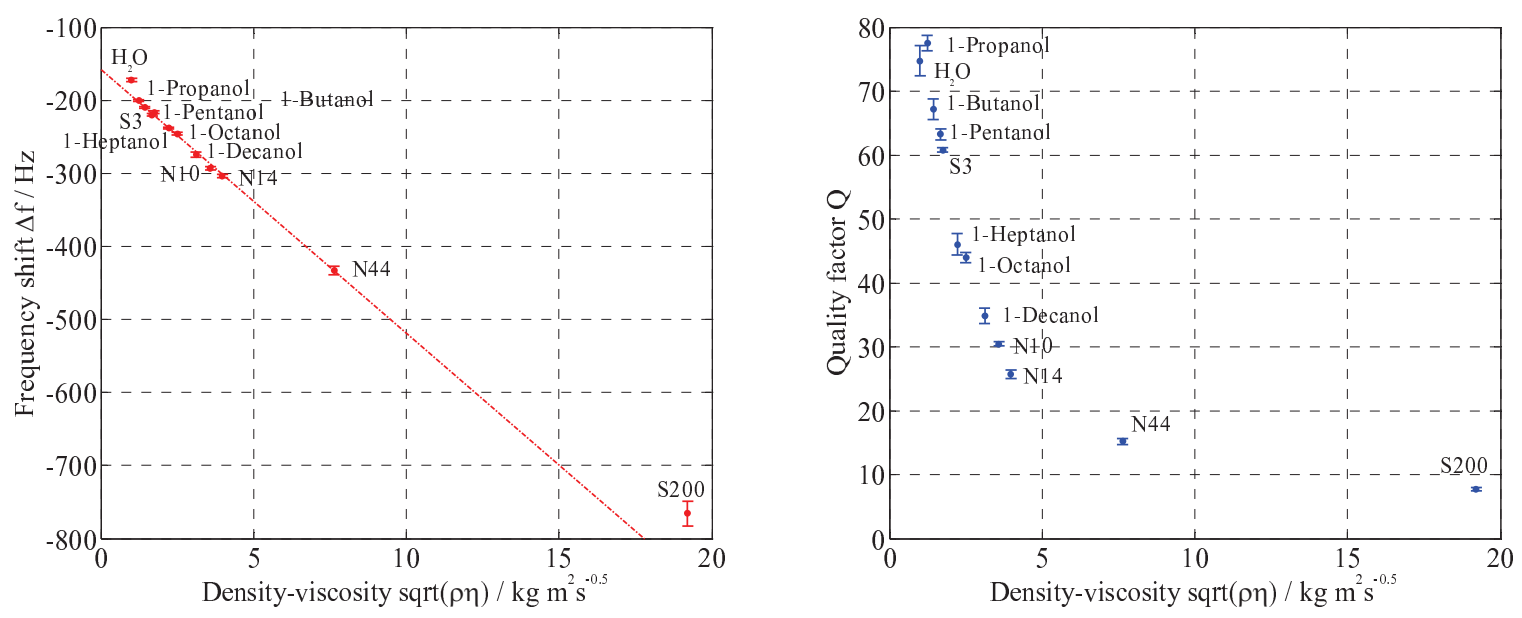

Fig. 3: Measurement of frequency shift (left) and Q-factor (right) vs. square root of density-viscosity product of different test liquids over range of $1-400 \mathrm{mPas}$ viscosity for $100 \mu \mathrm{m}$ nickel-zinc alloy plate resonator in horizontal vibration mode at $9 \mathrm{kHz}$, with mean value and standard deviation over 10 consecutive measurements. 


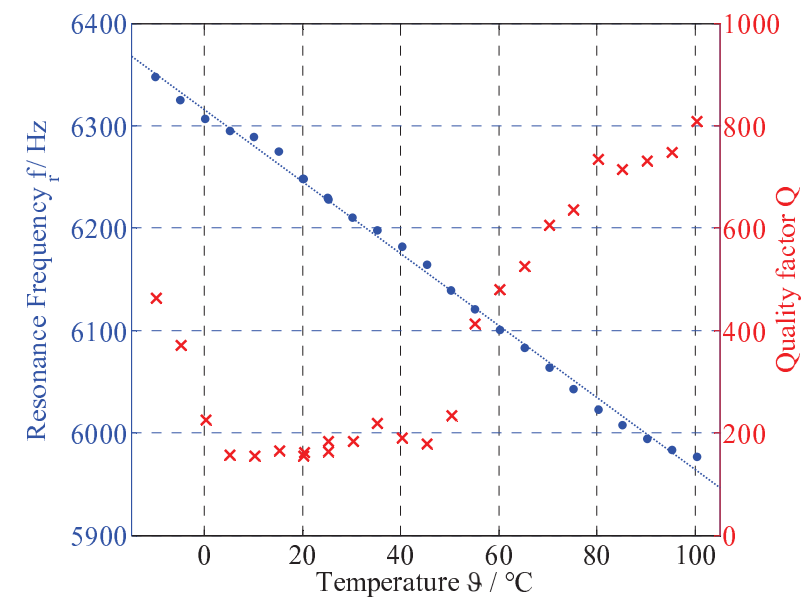

Fig. 4: Changes in Q-factor and frequency shift of $200 \mu \mathrm{m}$ thick stainless steel resonator operating in air over a temperature range of $-10-100^{\circ} \mathrm{C}$.

frequency shows a linear decrease. The quality factor however displays a curious minimum between 5 and $45^{\circ} \mathrm{C}$, while rising at lower and higher temperatures. The large and non-linear dependence of the quality factor on temperature due to changes in boundary conditions, elastic and geometric properties, and the electrical contacts suggest difficulties in separating resonator and setup temperature effects (both captured by this experiment).

To investigate this behavior we tested the stainless steel resonator in two different oil viscosity standards, S3 and N14 (Fig. 5). For increasing temperature the viscosity of the samples drops, corresponding to a reduction in viscous loading, which should result in an increase of resonance frequency and quality factor (see also Fig. 3). However, this is not reproduced in the measurements, where the resonance frequency decreases for increasing temperature (except for the low temperature region of the N14 oil measurements in Fig. 5 right). Given the fact that the device itself features a temperature dependence supporting this behavior (see Fig. 4), it can be concluded that this effect dominates the overall temperature coefficient (TC) of the frequency shift. Only for the sample N14 featuring a higher viscosity the sign of the overall TC is reversed at low temperatures $\left(<10^{\circ} \mathrm{C}\right)$. Therefore, determining the viscosity and density from the frequency shift requires a strict calibration.

In contrast, as quality factors due to different loss mechanisms can be added inversely and the $Q$ with liquid loading is roughly an order of magnitude below the quality factor of the unloaded device in air, we can conclude that the $Q$-factor is dominated by viscous damping. With increasing temperature and thus decreasing viscosity and density, the overall $Q$-factor increases, and can thus be utilized as a better measurement parameter for the density-viscosity product at different temperatures.
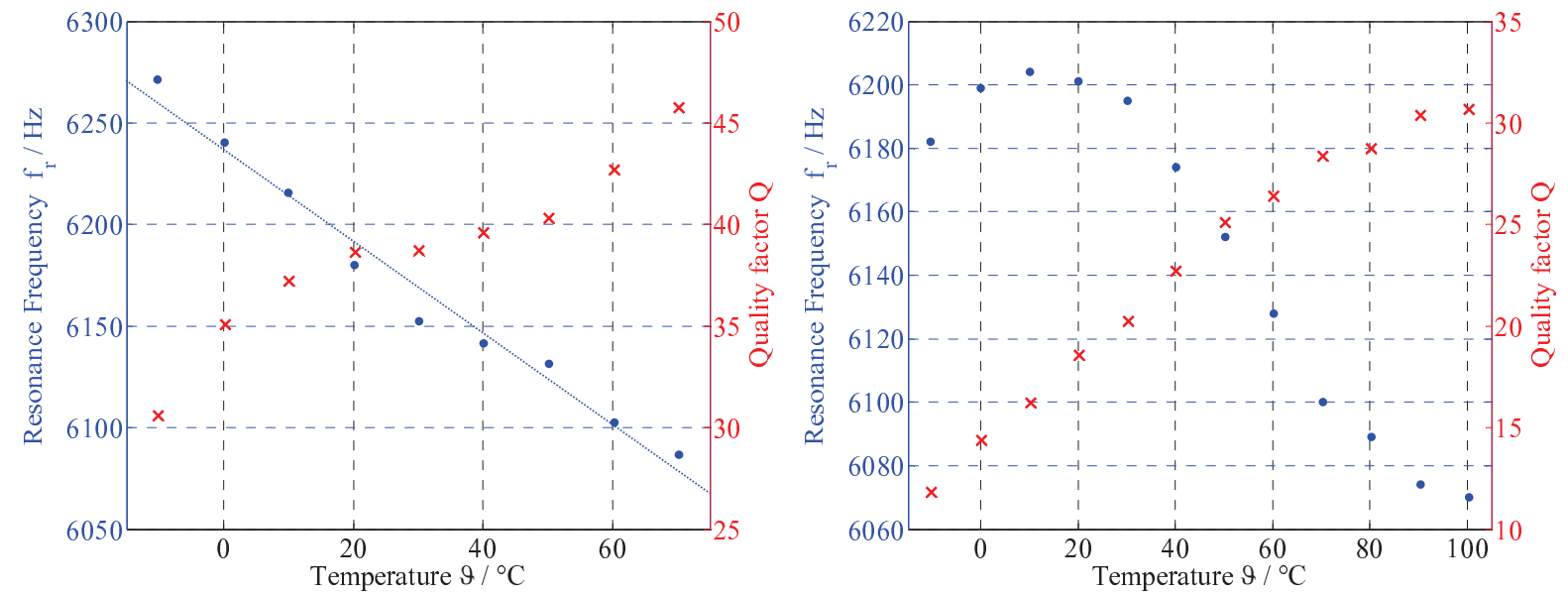

Fig. 5: Changes in Q-factor and frequency shift of $200 \mu \mathrm{m}$ thick stainless steel resonator operating in S3 viscosity standard (left) and N14 oil viscosity standard (right) over a temperature range of $-10-100^{\circ} \mathrm{C}$. 


\section{Summary and Conclusions}

We presented and evaluated a newly developed resonator sensor setup, based on electromagneticacoustic transduction. The sensor element, a suspended, conductive plate resonator, is clamped between multiple PCB layers, connected as a two-port device with individual excitation and readout paths, and vibrating in an in-plane, shear movement of the sensor surface. A small fluid measurement cell is defined into the PCB layers, requiring only minimal amounts of test liquid. The resonator response is analyzed in terms of quality factor and resonance frequency shift, which are, to first order, functions of the densityviscosity product. Measurement results show a very good correlation over a very wide range of different viscosities, with only minimal standard deviation over consecutive measurements for most liquids.

As a major challenge for applications it was found that the device shows a significant temperature dependence, which needs to be compensated if the resonance frequency is used as readout parameter. This problem is less pronounced for the quality factor, which is dominantly determined by the viscous damping.

With this sensor setup we have shown that microacoustic resonators can be employed as inexpensive density-viscosity sensors with reasonable precision requiring only a fraction of test liquid volume and analysis time compared to laboratory instruments.

\section{Acknowledgements}

This project is funded by the Austrian Center of Competence in Mechatronics (ACCM), Linz. We want to thank Bernhard Mayrhofer and Johann Katzenmayer for their support with lithography and fabrication of the sensor devices.

\section{References}

[1] M.J. Vellekoop, "Physical chemosensors," in Smart Sensor Systems, G.C.M. Meijer, Ed., ch5, New York: Wiley, (2008), pp. 121-150.

[2] B. Jakoby, R. Beigelbeck, F. Keplinger, F. Lucklum, A. Niedermayer, E.K. Reichel, C. Riesch, T. VoglhuberBrunnmaier, and B. Weiss, "Miniaturized sensors for the viscosity and density of liquids-performance and issues", IEEE Trans. Ultrason., Ferroel., Freq. Contr., vol. 57 (2010), pp. 111-120.

[3] C.D. Angelis, V. Ferrari, D. Marioli, E. Sardini, M. Serpelloni, and A. Taroni, "Magnetically induced oscillations on a conductive cantilever for resonant microsensors", Sensors Actuators A, vol. 135 (2007), pp.197-202.

[4] F. Lucklum, B. Jakoby, "Novel magnetic-acoustic resonator sensors for remote liquid phase measurement and mass detection", Sensors Actuators A, vol. 145/146 (2008), pp. 44-51.

[5] E.K. Reichel, C. Riesch, F. Keplinger, C.E.A. Kirschhock, B. Jakoby, "Analysis and experimental verification of a metallic suspended plate resonator for viscosity sensing", Sensors Actuators A, vol. 162 (2010), pp. 418-424.

[6] F. Lucklum, B. Jakoby, "Electromagnetic-acoustic high-Q silicon resonators for liquid phase sensing", IEEE Trans. Ultrason., Ferroel., Freq. Contr., vol. 57 (2010), pp. 1000-1002.

[7] K.K. Kanazawa, J.G. Gordon, "The oscillation frequency of a quartz resonator in contact with a liquid", Anal. Chim. Acta, vol. 175 (1985), pp. 99-105.

[8] S.J. Martin, V.E. Granstaff, G.C. Frye, "Characterization of a quartz crystal microbalance with simultaneous mass and liquid loading", Anal. Chem., vol. 63 (1991), pp. 2272-2281.

[9] S.V. Krishnaswamy, J. Rosenbaum, S. Horwitz, C. Vale, R.A. Moore, "Film bulk acoustic wave resonator technology", Proc. IEEE Ultrason. Symp., Honolulu, December 1990, pp. 529-536.

[10] X.C. Jin, I. Ladabaum, B.T. Khuri-Yakub, "The micro-fabrication of capacitive ultrasonic transducers", IEEE J. Microelectromech. Syst., vol. 7 (1998), pp. 295-302.

[11] C. Behling, R. Lucklum, P. Hauptmann, "Response of quartz-crystal resonators to gas and liquid analyte exposure", Sensors Actuators A, vol. 68 (1998), pp. 388-398.

[12] F. Herrmann, B. Jakoby, J. Rabe, S. Büttgenbach, "Microacoustic sensors for liquid monitoring", Sensors Update, vol. 9 (2001), pp. 105-160.

[13] G.G. Yaralioglu, M.H. Badi, A.S. Ergun, C. Cheng, B.T. Khuri-Yakub, F.L. Degertekin, "Lamb wave devices using capacitive micromachined ultrasonic transducers", Appl. Phys. Lett., vol. 78 (2001), pp. 111-113.

[14] P. Hauptmann, N. Hoppe, A. Püttmer, "Application of ultrasonic sensors in the process industry", Meas. Sci. Technol., vol. 13 (2002), pp. R73-R83.

[15] C. Vancura, Y. Li, K.U. Kirstein, F. Josse, A. Hierlemann, J. Lichtenberg, "Fully integrated CMOS resonant cantilever sensor for biochemical detection in liquid environments", Digest Tech. Papers Transducers'05, Seoul, Korea, June 2005, pp. 640-643. 
[16] C. Riesch, E.K. Reichel, A. Jachimowicz, J. Schalko, P. Hudek, B. Jakoby, and F. Keplinger, "A suspended plate viscosity sensor featuring in-plane vibration and piezoresistive readout", J. Micromech. Microeng., vol. 19 (2009), p. 075010.

[17] F. Lucklum, B. Jakoby, "Non-contact liquid level measurement with electromagnetic-acoustic resonator sensors", Meas. Sci. Technol., vol. 20 (2010), p. 124002. 